$10-5-2020$

\title{
Project SOAR research informs national policy change in Tanzania expanding access to HIV services for marginalized populations
}

Population Council

Follow this and additional works at: https://knowledgecommons.popcouncil.org/

series_newsletters_researchimpact

Part of the Health Services Research Commons, International Public Health Commons, and the Virus Diseases Commons

How does access to this work benefit you? Let us know!

\section{Recommended Citation}

"Project SOAR research informs national policy change in Tanzania expanding access to HIV services for marginalized populations," Research Utilization and Impact Brief. Washington, DC: Population Council, 2020. 


\title{
Project SOAR Research Informs National Policy Change in Tanzania Expanding Access to HIV Services for Marginalized Populations
}

\begin{abstract}
A recent Project SOAR study evaluated community-based delivery of antiretroviral treatment to female sex workers (FSWs) in Tanzania, demonstrating the value of this approach to improve HIV care. Informed by these findings, the Government of Tanzania changed national guidance to allow for community-based HIV treatment to better meet the needs of marginalized populations, including FSWs.
\end{abstract}

\section{INTRODUCTION}

Globally, female sex workers (FSWs) living with HIV are less likely ${ }^{1}$ to be on antiretroviral treatment (ART) than other populations; on average, fewer than 50 percent of FSWs living with HIV are on treatment. Research in Tanzania has documented the challenges $\mathrm{FSWs}^{2}$ face in accessing HIV care and treatment services, including out-of-pocket costs, reaching distant clinics, lack of awareness and misperceptions of treatment, and dual stigma associated with sex work and HIV status. Studies from sub-Saharan Africa have shown improved HIV treatment outcomes, such as uptake of HIV services, retention in care, and increased dignity and quality of life, by using community-based delivery of HIV services.

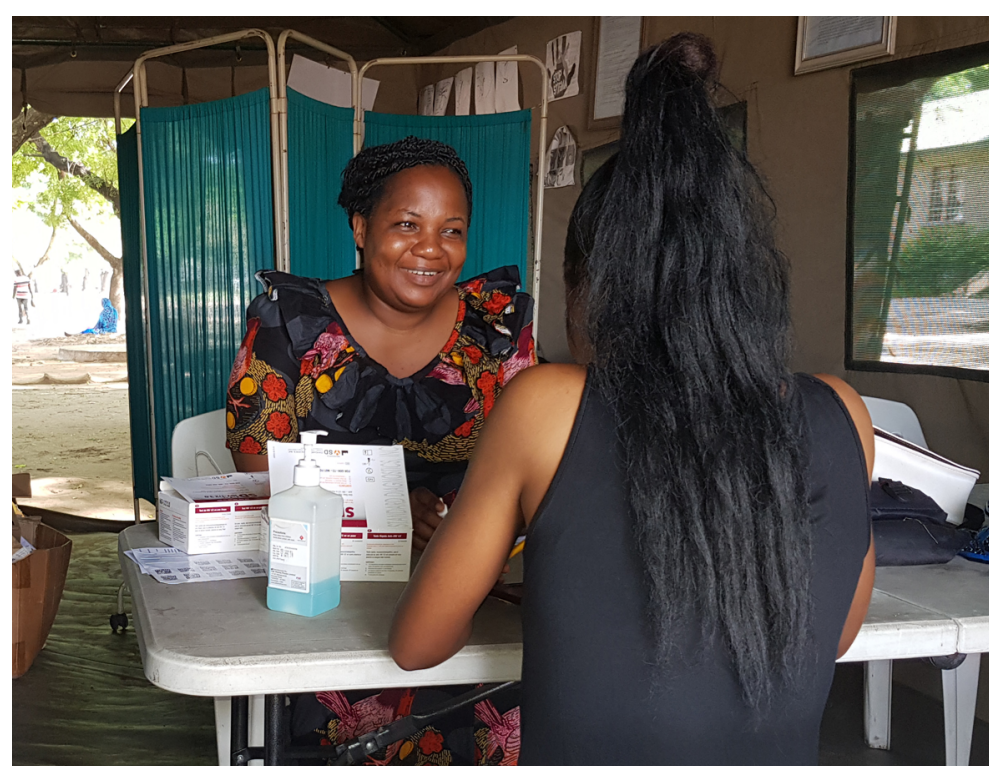

A female sex worker receives community-based ART services from a nurse. (Photo: @ CSK RESEARCH SOLUTIONS)
Between 2017 and 2019, Population Council's Project SOAR (Supporting Operational AIDS Research), ${ }^{3}$ funded by the US Agency for International Development, conducted an implementation science study to investigate community-based delivery of ART services to FSWs in Tanzania. This effort was undertaken in close collaboration with the National
AIDS Control Program (NACP) of the Government of Tanzania, National Institute of Medical Research Mwanza Research Centre, and Jhpiego's Sauti Program. The goal of the study was to understand if community-based ART delivery could help FSWs initiate and stay on HIV treatment. 


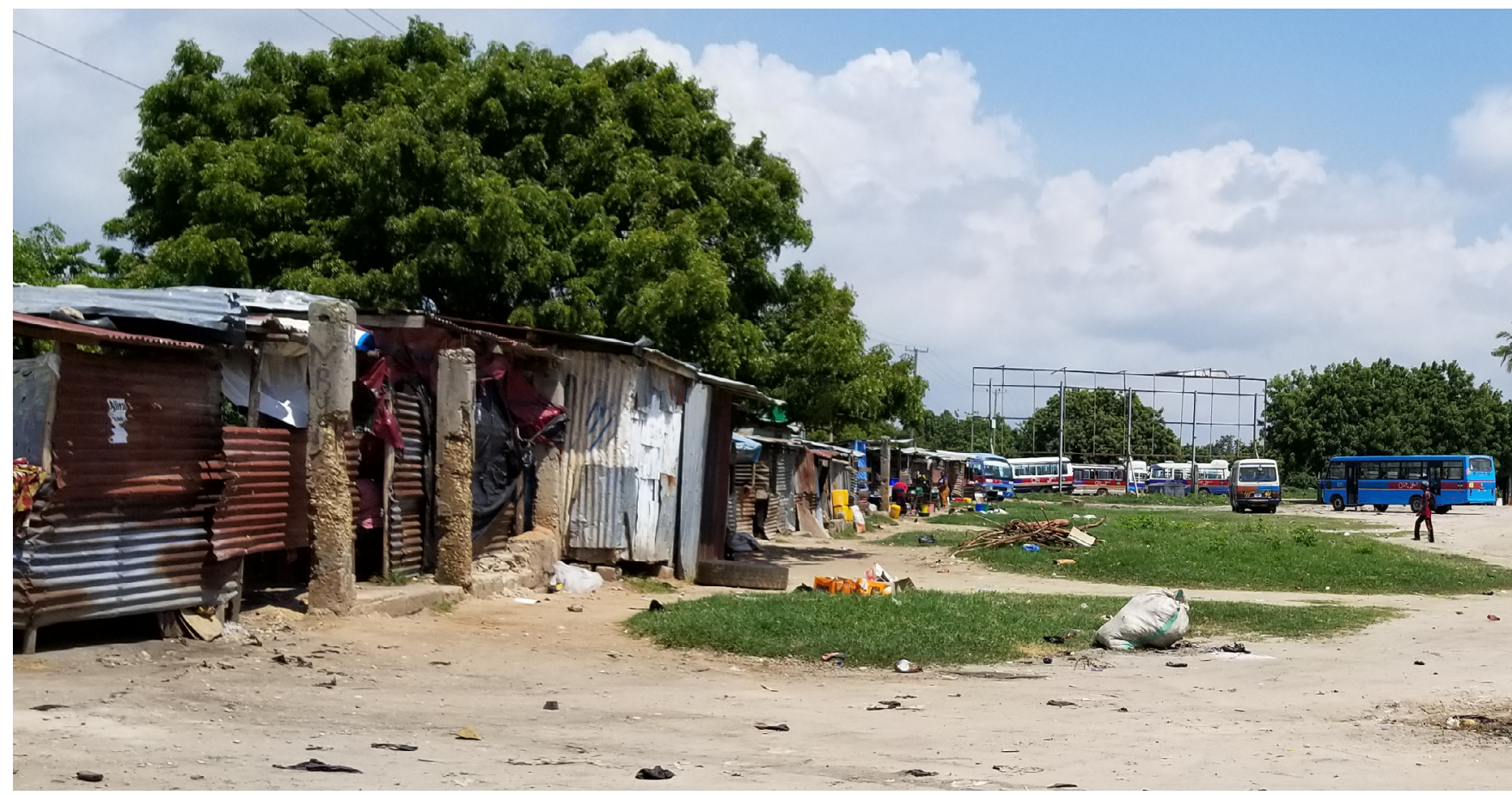

In understanding the impacts of community-based ART delivery, health providers offered care in locations where female sex workers felt most comfortable, including in their homes. (Photo: @ CSK RESEARCH SOLUTIONS)

\section{RESEARCH SUMMARY}

The project used a quasi-experimental prospective study design ${ }^{4}$ to explore the effects of a communitybased ART delivery model on three primary outcomes:

- Treatment initiation: Proportion of individuals linked to care and initiating ART treatment

- Retention in care: Proportion of individuals retained in care at 6 and 12 months after enrollment in care

- Adherence to treatment: Proportion of individuals adhering to ART measured by selfreporting and viral suppression at 6 and 12 months

The community-based ART service delivery model was built upon Sauti's existing community-based HIV testing and counseling plus ( $\mathrm{CBHTC}+$ ) intervention that provided additional services to key populations. Sauti operated in all seven study districts and together with the government provided the CBHTC+ package of services. In the intervention arm, ART was delivered through mobile community-based HIV testing and counseling services and home visits in four districts of the Njombe region. The comparison arm of the study was conducted in three districts of the Mbeya region where ART services were available through referrals to government-designated ART care and treatment clinics. A total of 617 FSWs were enrolled in the study.

Research findings ${ }^{5}$ demonstrated that FSWs in the community-based ART arm were more likely to initiate treatment than FSWs in the comparison arm. FSWs receiving communitybased ART also had higher retention rates, which remained high even after 12 months in the program. Conversely, the study found a significant drop off in participation for the comparison group between 6 and 12 months. Overall satisfaction with ART services was higher among FSWs receiving the community-based ART services, who spoke positively about client-provider interactions, information they received (adherence counseling, risk reduction), and perceived competence of the providers. However, the intervention had no effect on adherence or viral suppression, which were fairly high across both study arms. 


\section{KEY IMPACTS}

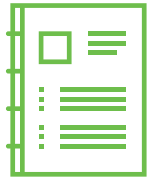

INFORMED BY PROJECT SOAR, NATIONAL GUIDANCE AND JOB AIDS UPDATED TO INCLUDE COMMUNITY-BASED DELIVERY OF ART TO REACH KEY AND VULNERABLE POPULATIONS

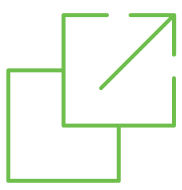

COMMUNITY-BASED ART SERVICES SUSTAINED IN STUDY SITES AND CONTINUING TO SCALE ACROSS TANZANIA WITH SUPPORT FROM MULTIPLE PARTNERS

\section{RESEARCH USE AND IMPACT}

Informed by the study findings, public facilities across Tanzania now provide community-based delivery of ART to reach key and vulnerable populations, including FSWs. Guidance on ART provision via mobile outreach was first included in the National Multisectoral Strategic Framework for HIV and AIDS $2018 / 19$ to $2022 / 23^{6}$ and is now featured in NACP's Operational Manual ${ }^{7}$ and Job Aids ${ }^{8}$ for Comprehensive Differentiated Delivery of HIV and AIDS Services.

In line with Project SOAR's approach to research utilization, ${ }^{8}$ the study was conceptualized and implemented in close collaboration with NACP and other partners to foster use of study results. Research responded to the needs of government officials and other partners to inform forthcoming guidance on decentralized delivery of HIV and AIDS services, specifically mobile outreach for marginalized populations. Throughout the research process, Project SOAR regularly shared data with the NACP, Sauti, the regional, district and community health management teams, and other partners through interim data workshops and formal presentations. Critically, NACP provided ongoing technical support, recommendations, and buy-in, the Njombe Regional Health Management Team (RHMT) conducted quarterly site visits and provided supervision for delivery of ART services, and facility CTC staff periodically joined Sauti ART delivery teams to observe and supervise patient visits.

Study results, along with data from other partners, reinforced that community-based ART provision was an effective and reliable model for improving initiation and retention in Tanzania, contributing to national policy and guideline changes. Highlighting the sustainability of this endeavor, community ART services remain available in study intervention districts within Njombe region, now managed by facility partners. Notably, these services also continue to scale in all regions across the country with support from USAID and CDC HIV service implementation partners. Beyond the Tanzanian context, this study has been cited in wider analyses ${ }^{9}$ of emergent service delivery priorities for global HIV programming.

For more information, please visit Project SOAR, which features additional resources documenting

its approach to research utilization employed

in Tanzania and more than 20 other countries worldwide.

\section{KEY RESOURCES}

Documenting Methods and Results to Support Robust HIV Service Delivery Among Female Sex Workers

Documenting Research Utilization Approaches to Promote Stakeholder Engagement and Research Impact
Full Report: Community-based HIV treatment service delivery model for female sex workers in Tanzania: Evaluation findings

Journal Article: Community-based antiretroviral therapy (ART) delivery for female sex workers in Tanzania: Intervention model and baseline findings Journal Article: Community-based antiretroviral therapy (ART) delivery for female sex workers in Tanzania: 6-month ART initiation and adherence

Results Brief: Community-based delivery of antiretroviral treatment for female sex workers in Tanzania: High levels of initiation, use, and adherence

Blog: Sam Kalibala, Director of Research Utilization, answers questions about SOAR's strategy to promote research uptake

Toolkit: Project SOAR's approach to research utilization 
${ }^{1,4}$ Community-based HIV treatment service delivery model for female sex workers in Tanzania: Evaluation findings. knowledgecommons. popcouncil.org/departments_sbsr-hiv/363/

2 Barriers and facilitators of retention in HIV care and treatment services in Iringa, Tanzania: the importance of socioeconomic and sociocultural factors. DOI: 10.1080/09540121.2013.861574

3 Project SOAR. projsoar.org/

5 Community-based antiretroviral therapy (ART) delivery for female sex workers in Tanzania: 6-month ART initiation and adherence.

DOI: 10.1007/s10461-019-02549-x
6 Tanzania national multisectoral strategic framework for HIV and AIDS 2018/19 to 2022/23. tacaids.go.tz/phocadownload/NMSF_IV2018.pdf

7 Operational manual for comprehensive differentiated delivery of HIV and AIDS services-December 2019. nacp.go.tz/download/tanzanianational-multisectoral-strategic-framework-for-hiv-and-aids-2018-19to-2022-23/

8 Job aids for comprehensive differentiated delivery of HIV and AIDS services. nacp.go.tz/download/job-aids-for-comprehensivedifferentiated-delivery-of-hiv-and-aids-services/

9 Emerging priorities for HIV service delivery. DOI: 10.1371/journal. pmed.1003028

Project SOAR (Cooperative Agreement AID-OAA-A-14-00060) is made possible by the generous support of the American people through the United States President's Emergency Plan for AIDS Relief (PEPFAR) and the United States Agency for International Development (USAID). The contents of this brief are the sole responsibility of Project SOAR and the Population Council and do not necessarily reflect the views of PEPFAR, USAID, or the United States Government.

The Population Council collaborates with program implementers, policymakers, researchers, and funding partners to advance evidence-based solutions to critical health and development challenges. The Council's research impact briefs feature recent highlights of our ongoing work to ensure evidence is translated into meaningful actions at community, national, regional, and global levels to improve lives around the world.

Recommended citation: "Project SOAR Research Informs National Policy Change in Tanzania Expanding Access to HIV Services for Marginalized Populations," Research Utilization and Impact Brief. Washington, DC: Population Council, 2020. 